\title{
Singapore's COVID-19 “circuit breaker" interventions: A description of individual-level adoptions of precautionary behaviours
}

\author{
Aidan Lyanzhiang $\underline{\operatorname{Tan}}{ }^{1} M B B S$, Sheryl Hui-Xian $\underline{\mathrm{Ng}}{ }^{1} M P H$, Michelle Jessica Pereira ${ }^{1}{ }_{P h D}$
}

\begin{abstract}
Introduction: Effectiveness of COVID-19 control interventions relies significantly on behavioural modifications of its population. Differing adoption rates impacts subsequent COVID-19 control. Hence, positive and sustained behavioural modification is essential for disease control. We describe the adoption rates of behavioural modifications for Singapore's "circuit-breaker" (CB), the national public health response to the COVID-19 crisis, among the general population in the community.

Methods: We conducted an interrupted-time series study using retrospective secondary data. We compared the proportion of Singaporeans who reported adopting specific behaviour modifications before, during and after $\mathrm{CB}$. Behaviours of interest were working from home, performing hand hygiene, using face mask in public, and avoiding crowded areas. We compared change in incidence rates for community COVID-19 cases among the general population across the same time periods.

Results: There was an increase in face mask usage $(+46.9 \%, 95 \%$ confidence interval $[\mathrm{CI}] 34.9-58.8$, $P<0.01)$ and working from home $(+20.4 \%, 95 \%$ CI $11.7-29.2, P<0.01)$ during $\mathrm{CB}$ than before CB in Singapore. Other self-reported behaviours showed no statistically significant difference. Change in daily incidence rates of community COVID-19 cases decreased from additional 0.73 daily case before $\mathrm{CB}$ to 0.55 fewer case per day during $\mathrm{CB}(P<0.01)$. There was no significant difference among all behaviour adoption rates after $\mathrm{CB}$. Daily incidence of community cases continued to decrease by 0.11 case daily after $\mathrm{CB}$.

Conclusion: Community incidence of COVID-19 in Singapore decreased during CB and remained low after $\mathrm{CB}$. Use of face masks and social-distancing compliance through working from home increased during CB. However, it is unlikely to influence other sources of COVID-19 such as imported cases or within foreign worker dormitories.
\end{abstract}

Ann Acad Med Singap 2021;50:613-8

Keywords: Behaviours, COVID-19, public health

\section{INTRODUCTION}

In response to the COVID-19 pandemic, many countries have adopted similar public health measures such as enforced social distancing via lockdowns or universal face mask usage..$^{1-5}$ However, population compliance with these behavioural interventions varies, likely due to differences in intervention, implementation and country characteristics. ${ }^{1-3,5}$ The differing compliance levels resulted in varying effectiveness of COVID-19 control despite similarity in interventions. ${ }^{6}$ In South Korea, one of the key factors in the country's effective control of COVID-19 was good public acceptance and compliance with COVID-19 control measures. ${ }^{5}$ In contrast, despite similar strategies employed in the UK, public unreceptivity and non-compliance in the community resulted in lack of effectiveness. ${ }^{7}$ Therefore it is important to measure and encourage recipient support and compliance with the interventions to ensure effective disease control.

The first COVID-19 case in Singapore was reported in February 2020. ${ }^{8}$ Daily reported incidence of local community cases increased from single-digit counts in early March to more than 30 in the first week of April. This was despite border control measures

${ }^{1}$ Health Services and Outcomes Research, National Healthcare Group, Singapore

Correspondence: Dr Aidan Lyanzhiang Tan, Health Services and Outcomes Research (HSOR), National Healthcare Group, 3 Fusionopolis Link, \#03-08, Nexus@one-north, Singapore 138543.

Email: aitan@nhg.com.sg 


\section{CLINICAL IMPACT}

\section{What is New}

- Community COVID-19 incidence in Singapore decreased during circuit breaker and remained low after.

- Use of face masks and social-distancing compliance increased during circuit breaker.

\section{Clinical Implications}

- Positive and sustained behavioural modifications are essential for COVID-19 control.

and social-distancing advisories. ${ }^{8} \mathrm{~A}$ nationwide containment intervention or "circuit breaker" (CB) was thus enacted from 7 April to 1 June 2020. As many countries experienced resurgences post-lockdown, ${ }^{3,5}$ Singapore's lockdown was gradually relaxed in phases. Further details are available in the literature. ${ }^{9-11}$

Singapore's CB measures included various forms of mandatory behavioural modifications with legal penalties such as fines. However, individual compliance with these specified behaviours has varied even with legal mandates. A cross-sectional study in Ghana described only $18.9 \%$ adoption of face masks on public transport despite it being mandatory at the time of the study. ${ }^{12}$ In contrast, a video-analysis study showed compliance with mandatory face mask usage as $85.5 \%$ in South Korea and $99.4 \%$ in China. ${ }^{13}$ To our knowledge, there is a lack of descriptive literature on Singapore's behavioural modification adoption rates. This is worrying particularly as there have been anecdotal reports of non-compliance in the news media.

We describe the effectiveness of Singapore's public health response to the COVID-19 crisis, looking at adoption rates of behavioural modification and other public health measures.

\section{METHODS}

\section{Design}

We conducted an interrupted time-series study to retrospectively evaluate Singapore's national COVID-19 interventions. Our study population was the general population residing in the community, not including foreign workers or imported cases.

\section{Outcomes}

\section{Behavioural modifications}

As part of the CB measures, all non-essential workplaces and organisations were mandated to close or implement work-from-home arrangements. As directly measured data on the number of organisations implementing work-from-home arrangements were not available, we used a proxy downstream measure (proportion of workers that reported being able to work from home) although this was less than ideal.

Other behavioural modifications were self-reported compliance with face mask-wearing in public areas, personal hygiene via handwashing or hand sanitiser use, and avoidance of crowded areas. This was derived from secondary data on Singaporeans via an online survey conducted by Imperial College and YouGov Plc. ${ }^{14}$

\section{Change in local community COVID-19 incidence}

We collected daily reported local COVID-19 cases (daily incidence) as reported by the Ministry of Health Singapore $(\mathrm{MOH})$ and compared the change in daily incidence (incidence growth or slope).

\section{Maintenance of behavioural modifications}

We compared the above-mentioned outcomes between the periods during and after CB. Singapore had a graded approach to release of $\mathrm{CB}$ restrictions, termed as Phase 1 (2-19 June 2020) and Phase 2 (20 June 2020-28 December 2020). For simplicity of assessment and due to the short duration of these phases, we combined both into a single time period of "after CB".

\section{Data sources}

For disease incidence, we used publicly available COVID-19 press release data from MOH. Disease counts were obtained from mandatory nationwide reporting by doctors and clinical laboratories to $\mathrm{MOH}$.

We used publicly available aggregate anonymised survey data on Singaporeans' self-reported behaviours to measure adoption of behavioural modifications. Survey questions were developed by the Institute of Global Health Innovation at Imperial College London. ${ }^{14}$ The weekly cross-sectional surveys were conducted online by YouGov Plc internationally. Survey samples were stated to be representative of the general public, based on age, gender and within-country regions. Approximately 1,000 Singaporeans were 
invited to complete the surveys every week from 21 February 2020 to 1 May 2020, and on a biweekly basis thereafter. Financial incentives by YouGov Plc were provided via points accrual from survey completion (SGD25 for 5,000 points). Each completed survey awarded participants approximately 400 points. Response rates were $99 \%$ or higher for survey questions relevant to our study.

Survey respondents were asked their frequency of certain behaviours in response to COVID-19 over the past week. Behaviours were: avoiding crowded public areas, performing handwashing or using hand sanitiser, using face masks in public areas, and avoiding of workplaces (or working from home). Responses were categorised into binary outcomes of compliant ("frequently" or "always") or not-compliant ("sometimes", "rarely" or "not at all"). Data is publicly available from the Imperial College London YouGov Covid 19 Behaviour Tracker Data Hub data repository (https://github.com/YouGov-Data/covid-19-tracker).

\section{Statistical analysis}

Data were categorised according to 3 time periods: (1) before CB (1 January-6 April 2020), (2) during CB (7 April-1 June 2020), and (3) after CB (2 June-5 August 2020), with the exception of COVID-19 incidence. We anticipated the CB to affect secondary transmission. Hence, we applied a 14-day (twice the average incubation period) lag after the start and end of $\mathrm{CB}$ for comparisons of COVID-19 incidence between the time periods.

Data were summarised and presented as proportions, counts or means with standard deviations as appropriate.

Segmented regression was used for slope and level comparisons across time periods. We tested for autocorrelation and non-stationarity in the data, ${ }^{15}$ and reported confidence intervals using Newey-West standard errors wherever correction for autocorrelation was required. ${ }^{16}$ All statistical analyses were carried out in R Version 4.0.2.

\section{Triangulation}

As these outcomes were drawn from separate noncombinable datasets and there is a risk of bias if analysed together, we only visually compared trends between outcomes and qualitatively assessed whether they were coherent with our hypothesis. As this study used secondary publicly available data, no ethics or institutional review board approval was required.

\section{RESULTS}

\section{Work-from-home arrangements}

The average proportion of Singaporeans reporting work-from-home arrangements was $17 \%(11-31 \%)$ prior to the $\mathrm{CB}$. There was a statistically significant increase during the $\mathrm{CB}(20.4 \%, 95 \%$ confidence interval $[\mathrm{CI}] 11.7-29.2, P<0.01)$, compared to before $\mathrm{CB}$ (Table 1). There was no statistically significant difference between periods during and after $\mathrm{CB}$.

\section{Face mask usage, handwashing, and avoiding crowded areas}

Before $\mathrm{CB}$, the proportion of individuals wearing face masks in public was on average $25 \%$ (standard deviation [SD] 5.4\%). During the $\mathrm{CB}$, it increased to $86 \%$ (SD 7.7\%), as shown in Fig. 1A. The difference in average proportion before and during $\mathrm{CB}$ was statistically significant $(46.9 \%, 95 \%$ CI $34.9-58.8$, $P<0.01)$. There were no significant changes in the other self-reported behaviours (Figs. 1B and 1C). Individuals reported a high tendency to avoid crowded public areas even prior to the $\mathrm{CB}(69 \%$, SD $12 \%, P=0.80)$ compared to during $\mathrm{CB}(85 \%$, SD $1.1 \%, P=0.80)$, and for performing handwashing or hand sanitiser use $(83 \%$, SD $3.2 \%$ vs $84 \%$, SD $0.8 \%, P=0.48)$. These behaviours remained high with no significant difference after CB (Table 1).

\section{Local community COVID-19 incidence}

Before $\mathrm{CB}$, the incidence growth rate was positive $(0.73, \mathrm{SD} 0.05)$, indicating an increasing incidence of 0.73 local case in the community day by day. During $\mathrm{CB}$, the incidence growth rate became negative $(-0.55$, SD 0.07), reflecting a daily decrease in incidence. The difference in growth rate between the two periods was significant $(-1.28,95 \% \mathrm{CI}-1.48$ to $-1.08, P<0.01)$. After $\mathrm{CB}$, the change in daily incidence of cases remained negative but at a gentler gradient (-0.11, SD 0.06; change in slope: $0.44,95 \%$ CI 0.26 to $0.62, P<0.01$ ), as shown in Fig. 2.

\section{DISCUSSION}

Behavioural modifications (proportion of Singaporeans wearing face masks in public, and those reporting work-from-home arrangements) showed an increase between the periods before $\mathrm{CB}$ and during $\mathrm{CB}$. The increase is likely due to these behavioural modifications being made mandatory. In Singapore, "social-distancing ambassadors" were deployed to 
Table 1. Reported adherence to Singapore's “circuit breaker" measures

\begin{tabular}{lccccc}
\hline & \multicolumn{2}{c}{$\begin{array}{c}\text { Proportion self-reported } \\
\text { behaviour }\end{array}$} & & $\begin{array}{c}\text { Change between periods, } \\
\text { level change estimate (95\% confidence interval) }\end{array}$ \\
\hline Time period relative to circuit breaker & Before & During & After & Before versus during & During versus after \\
\hline Wearing face masks in public, \% (SD) & $25.4(5.4)$ & $86(7.7)$ & $90.2(1.8)$ & $\mathbf{4 6 . 9 ~ ( 3 4 . 9 ~ t o ~ 5 8 . 8 ) ~}$ & $12.7(-8.8$ to 34.3) \\
\hline Avoiding crowded public areas, \% (SD) & $68.7(12.1)$ & $84.6(1.1)$ & $80(3.2)$ & $-1.6 \%(-15.3$ to 12.2) & $3.4(-8.0$ to 14.8) \\
\hline Performing handwashing or hand sanitiser use, \% (SD) & $82.6(3.3)$ & $83.8(0.8)$ & $80.4(1.3)$ & $-1.9(-7.8$ to 4.0) & $-3.6(-10.2$ to 2.9) \\
\hline Working from home, \% (SD) & $17.3(7.5)$ & $51.4(3.2)$ & $38.9(7.7)$ & $\mathbf{2 0 . 4 ~ ( 1 1 . 7 ~ t o ~ 2 9 . 2 ) ~}$ & $15.2(-3.5$ to 34.0) \\
\hline
\end{tabular}

${ }^{a}$ Confidence interval reported using Newey-West standard errors

Bold numbers are statistically significant

actively remind non-compliant members of the public to comply with face masks or social distancing. Punitive measures were also included in the legislation with strict enforcement.

We found a slope-change of the daily incidence of COVID-19 cases from positive (increasing daily incidence) to negative (decreasing daily incidence) during the $\mathrm{CB}$. Our study estimated the lockdown effect at negative growth rate of -1.28 cases daily. This was in line with other studies, where lockdowns reduced case incidence growth rates. ${ }^{6,17}$

When the $\mathrm{CB}$ was lifted in a phased manner, there was a continued but lesser reduction in daily incidence growth rate compared to during the $\mathrm{CB}$. This doseresponse relationship lends further support to the causal-attribution relationship between the $\mathrm{CB}$ and the observed outcomes. All behavioural modifications remained stable and did not differ significantly between the periods during and after $\mathrm{CB}$. This is likely due to the measured behaviours continued to be mandatory or public health recommendations.

Given these findings in adoption rates of behavioural modification and community COVID-19 incidence, we can assume that the CB was effective in controlling transmission in the general Singapore population in the community (outside the foreign worker dormitories). Some of the drivers for the CB's effectiveness were: enforced social distancing via mandatory work from home or suspension of services for all non-essential businesses, and universal face mask usage. These were outcomes that showed a significant increase in compliance and were also documented as effective containment measures in the literature..$^{1,4,6,17-21}$ However, our results only show an association between the intervention component and its effect; there was insufficient strength for causality given the crosssectional nature of data and the difficulty in comparability between datasets.
Our study focused on the general population in the community. However, a large proportion of Singapore's COVID-19 cases occurred due to the outbreak in foreign worker dormitories early into and during the $\mathrm{CB}$. This was due to multiple factors, including high population density and poor living conditions. Additional measures were required to control the outbreak. ${ }^{22,23}$ Thus, while we found $\mathrm{CB}$ to be effective in controlling community cases among the general population, these may be unlikely to influence other sources of COVID-19 such as imported cases or within the foreign worker dormitories.

Our study has some limitations. In epidemics, one of the ideal outcome measures for interventions is the effect on secondary transmissions. These include estimations of reproduction number (Rt) based on the infectious profile of the disease and parameters such as serial interval distribution, duration and proportion of asymptomatic spread. ${ }^{24,25}$ Given our limited data, coupled with the large proportion of asymptomatic infections for COVID-19, this would have required multiple assumptions and resulted in potentially unreliable estimates. Hence, while not ideal, we only analysed incidence pre and post $\mathrm{CB}$ to reflect the impact of these interventions on the spread of cases.

We had chosen to fit simple linear models across different time periods for simplicity of analysis and ease of interpretation of effects as averages for each period. Admittedly, this model is not fully representative of the complexities in reality. Other models such as those with quadratic or cubic effects, while providing results that are less straightforward in interpretation, would more fully characterise the trends being studied.

We used secondary cross-sectional data from multiple sources. These datasets were not combinable, making study of temporal associations on an individual level difficult. There may be bias in comparing trends due to differences in the sampled populations across time and between data sources. Hence, we were only able to 


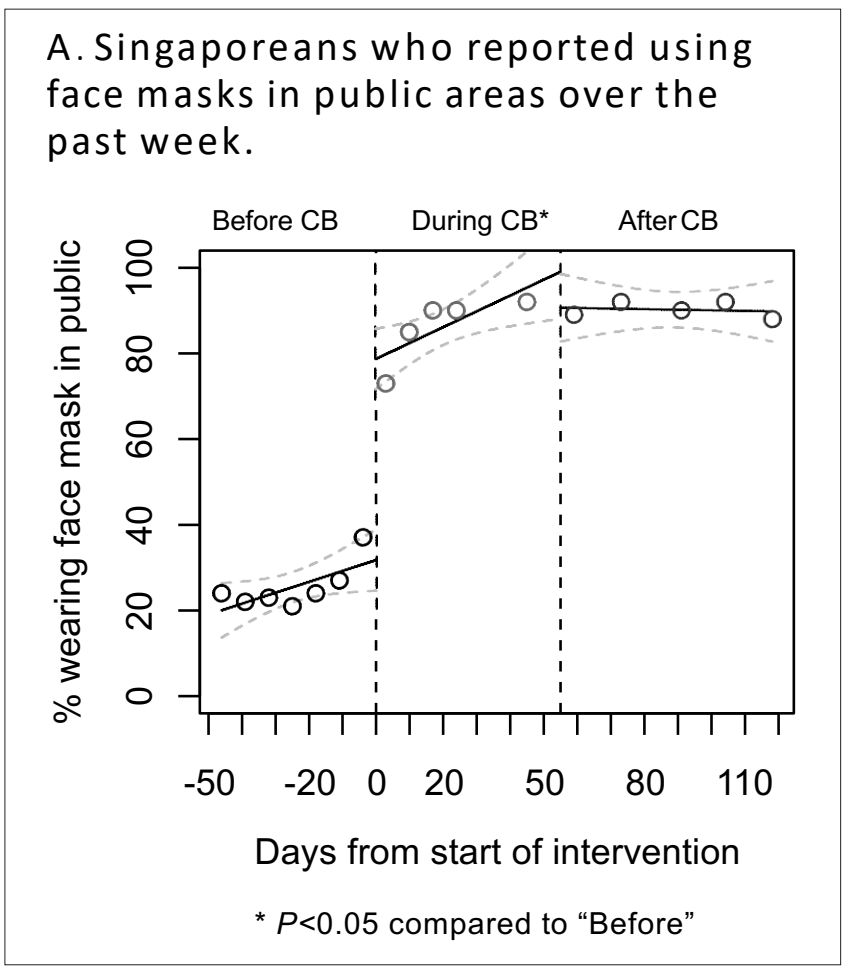

C. Singaporeans who reported washing hands or using hand sanitiser over the past week.

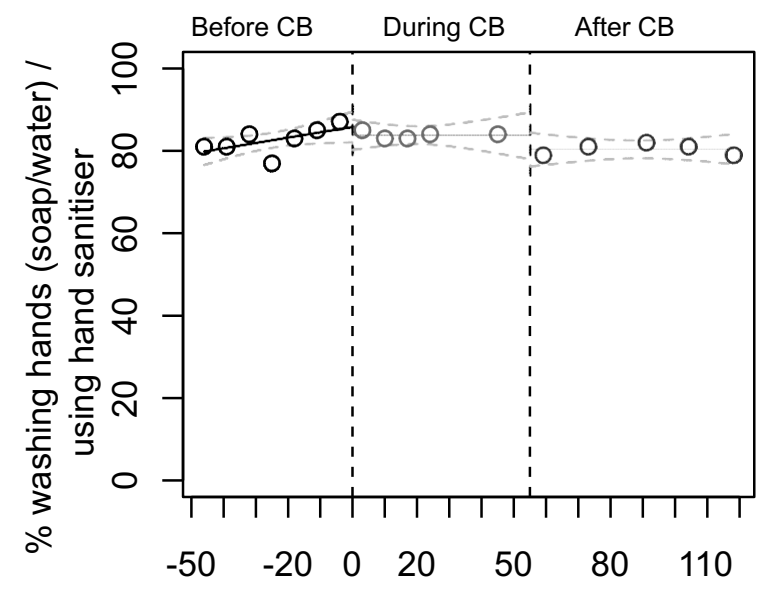

Days from start of intervention

\section{B. Singaporeans who reported avoiding crowded places over the past week.}

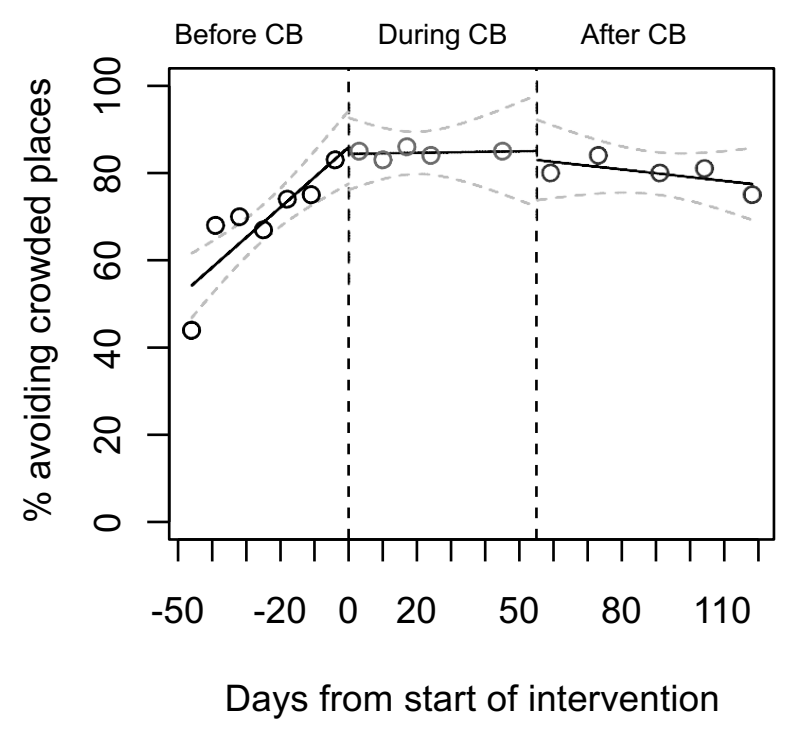

D. Singaporeans who reported work-from-home arrangements over the past week.

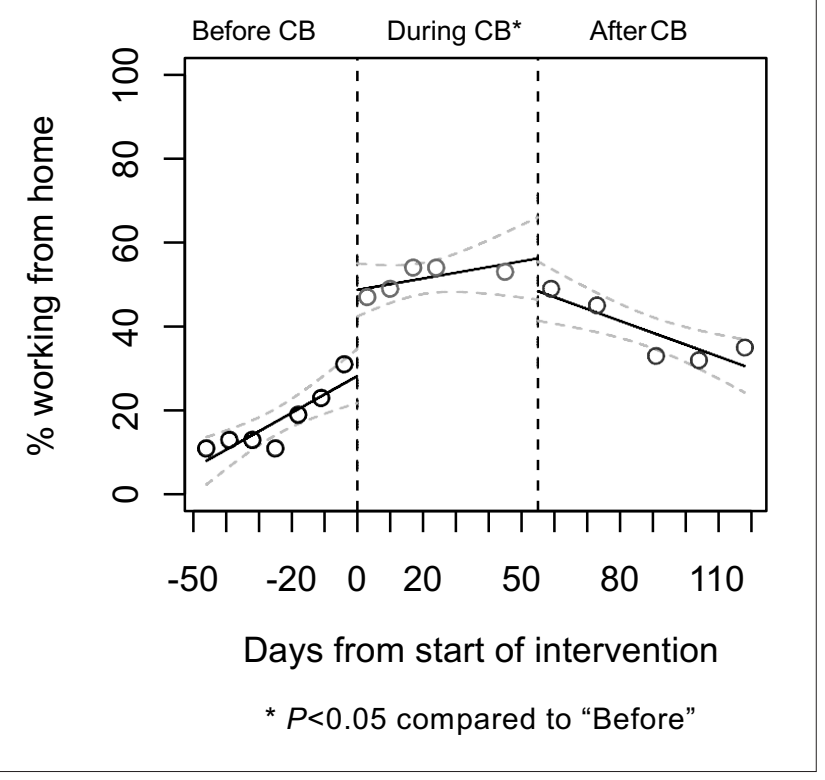

of $\mathrm{CB}$ interventions and identify a dose-response relationship. Our findings, while showing similar results to that in the literature, further builds on it by providing longer temporal view across periods of varying degrees 


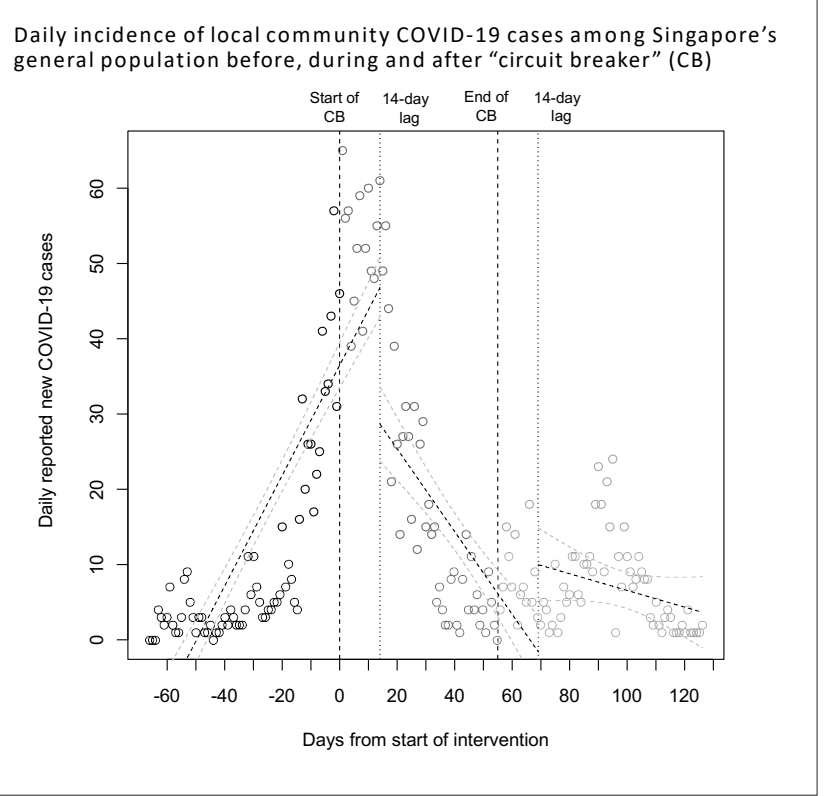

Fig. 2. Incidence of community COVID-19 in Singapore.

of interventions. We recommend further longitudinal cohort research at an individual level to determine the association between behavioural modification and observed COVID-19 control.

\section{CONCLUSION}

Community COVID-19 incidence in Singapore decreased during $\mathrm{CB}$ and remained low after $\mathrm{CB}$. Usage of face masks and social-distancing compliance through working from home increased during CB. However, it is unlikely to influence other sources of COVID-19 such as imported cases or within foreign worker dormitories.

\section{REFERENCES}

1. Ghosal S, Bhattacharyya R, Majumder M. Impact of complete lockdown on total infection and death rates: A hierarchical cluster analysis. Diabetes Metab Syndr 2020;14:707-11.

2. Atalan A. Is the lockdown important to prevent the COVID-9 pandemic? Effects on psychology, environment and economyperspective. Ann Med Surg (Lond) 2020;56:38-42.

3. Tobías A. Evaluation of the lockdowns for the SARS-CoV-2 epidemic in Italy and Spain after one month follow up. Sci Total Environ 2020;725:138539.

4. Leung NHL, Chu DKW, Shiu EYC, et al. Respiratory virus shedding in exhaled breath and efficacy of face masks. Nat Med 2020;26:676-80.

5. Oh J, Lee JK, Schwarz D, et al. National response to COVID-19 in the Republic of Korea and lessons learned for other countries. Health Syst Reform 2020;6:e1753464.
6. Islam N, Sharp SJ, Chowell G, et al. Physical distancing interventions and incidence of coronavirus disease 2019: Natural experiment in 149 countries. BMJ 2020;370:m2743.

7. Hirschhorn L, Smith JD, Frisch MF, et al. Integrating implementation science into covid-19 response and recovery. BMJ 2020;369:m1888.

8. Tariq A, Lee $\mathrm{Y}$, Roosa $\mathrm{K}$, et al. Real-time monitoring the transmission potential of COVID-19 in Singapore, March 2020. BMC Med 2020;18:166.

9. Chen JI, Yap JC, Hsu LY, et al. COVID-19 and Singapore: From early response to circuit breaker. Ann Acad Med Singap 2020;49: 561-72.

10. Chua AQ, Tan MMJ, Verma M, et al. Health system resilience in managing the COVID-19 pandemic: Lessons from Singapore. BMJ Global Health 2020;5:e003317.

11. Tan THY, Toh M, Vasoo S, et al. Coronavirus disease 2019 (COVID-19): The Singapore experience. A review of the first eight months. Ann Acad Med Singap 2020;49:764-78.

12. Dzisi EKJ, Dei OA. Adherence to social distancing and wearing of masks within public transportation during the COVID-19 pandemic. Transportation Research Interdisciplinary Perspectives 2020;7:100191.

13. Chen YJ, Qin G, Chen J, et al. Comparison of face-touching behaviors before and during the coronavirus disease 2019 pandemic. JAMA Netw Open 2020;3:e2016924.

14. Imperial College London and YouGov. COVID-19 public monitor. Available at: https://yougov.co.uk/covid-19. Accessed on 27 August 2020.

15. Wagner AK, Soumerai SB, Zhang F, et al. Segmented regression analysis of interrupted time series studies in medication use research. J Clin Pharm Ther 2002;27:299-309.

16. Linden A. Conducting interrupted time-series analysis for singleand multiple-group comparisons. The Stata Journal 2015;15:480-500.

17. Lonergan M, Chalmers JD. Estimates of the ongoing need for social distancing and control measures post-"lockdown" from trajectories of covid-19 cases and mortality. Eur Respir J 2020;56.

18. Esposito S, Principi N, Leung CC, et al. Universal use of face masks for success against COVID-19: Evidence and implications for prevention policies. Eur Respir J 2020;55:2001260.

19. Wei WE, Li Z, Chiew CJ, et al. Presymptomatic transmission of SARS-CoV-2 - Singapore, January 23-March 16, 2020. MMWR Morb Mortal Wkly Rep 2020;69:411-5.

20. Keshtkar-Jahromi M, Sulkowski M, Holakouie-Naieni K. Public masking: An urgent need to revise global policies to protect against COVID-19. Am J Trop Med Hyg 2020;102:1160-1.

21. Wilder-Smith A, Freedman DO. Isolation, quarantine, social distancing and community containment: Pivotal role for old-style public health measures in the novel coronavirus (2019-nCoV) outbreak. J Travel Med 2020;27:taaa020.

22. Koh D. Migrant workers and COVID-19. Occup Environ Med 2020;77:634-6.

23. Chew MH, Koh FH, Wu JT, et al. Clinical assessment of COVID-19 outbreak among migrant workers residing in a large dormitory in Singapore. J Hosp Infect 2020;106:202-3.

24. Thompson RN, Stockwin JE, van Gaalen RD, et al. Improved inference of time-varying reproduction numbers during infectious disease outbreaks. Epidemics 2019;29:100356.

25. Cori A, Ferguson NM, Fraser C, et al. A new framework and software to estimate time-varying reproduction numbers during epidemics. AmJ Epidemiol 2013;178:1505-12. 